\title{
Esophageal Neuroendocrine Tumor G2
}

National Cancer Institute

\section{Source}

National Cancer Institute. Esophageal Neuroendocrine Tumor G2. NCI Thesaurus. Code C95617.

A well differentiated, intermediate grade tumor with neuroendocrine differentiation that arises from the esophagus. The mitotic count is 2-20 per $10 \mathrm{HPF}$ and/or the Ki67 index is 3 to 20 percent. 